\title{
Aprendizaje significativo con estrategia de enseñanza activa para un curso de proyecto software. Una experiencia en el norte de Chile
}

\author{
Meaningful learning with active teaching strategy \\ for a software project course. An experience in northern Chile \\ Víctor Flores ${ }^{1 *}$ \\ Recibido 5 de agosto de 2020, aceptado 16 de octubre de 2020 \\ Received: August 5, 2020 Accepted: October 16, 2020
}

\begin{abstract}
RESUMEN
En la enseñanza de asignaturas relativas a proyectos software, la incorporación de metodologías activas ha traído buenos resultados en carreras de ingeniería en Informática y Computación. Durante los últimos 20 años, la metodología Aprendizaje más Servicio $(\mathrm{A}+\mathrm{S})$ ha contribuido significativamente con estos resultados. Este artículo presenta los resultados de una estrategia de Metodología Activa en la asignatura Taller de Programación de la carrera Ingeniería de Ejecución en Computación e Informática. En la estrategia, la metodología $\mathrm{A}+\mathrm{S}$ ha sido usada en un desarrollo software solidario, orientado a dar soporte a la comunidad. Usando esta estrategia, competencias como diseño e implementación de software con programación orientada a objetos y calidad software han sido desarrolladas. También se ha orientado el trabajo para desarrollar otras competencias como trabajo en grupo, comunicación oral y escrita y autogestión, todas estas necesarias para el correcto desarrollo profesional del estudiante del siglo XXI.
\end{abstract}

Palabras clave: Estrategia de enseñanza activa, aprendizaje significativo, proyectos software.

\begin{abstract}
In the teaching of subjects related to software projects, the incorporation of active learning methodologies has brought good results in computer science and computing engineering careers. During the last 20 years, the Learning plus Service $(A+S)$ methodology has contributed significantly to these results. This article describes the results of an active teaching strategy in the Programming Workshop subject of the Technical Engineering in Computing and Informatics career. In the strategy, the A $+S$ methodology has been used for a solidary software development, in order to giving support to the community. Using this strategy, skills such as software design and implementation with object-oriented programming and software quality have been developed. The work has also been oriented to develop other skills such as group work, oral and written communication and self-management, all these skills necessary for the correct professional development of the 21 st century.
\end{abstract}

Keywords: Active learning methodologies, meaningful learning, software projects.

\footnotetext{
1 Universidad Católica del Norte. Departamento de Ingeniería de Sistemas y Computación. Antofagasta, Chile.

* Autor de correspondencia: vflores@ucn.cl
} 


\section{INTRODUCCIÓN}

En la década de 1990 el Espacio Europeo de Educación estableció desafíos importantes con el objetivo de generar cambios significativos en la enseñanza [1]. En Latinoamérica y concretamente en proyectos software, se han asumido también desafíos con objetivos claros, como por ejemplo: mejorar el aprendizaje de metodologías de desarrollo de proyectos software. Casos concretos son [2,3], que describen trabajos para disminuir la brecha entre lo que se hace en las salas de clase y lo que se requiere en las empresas.

En Chile se están desarrollando proyectos comunitarios solidarios desde 2016, en áreas como educación, salud, bienestar público, obras públicas, medioambiente, agricultura y desastres naturales. Esto mediante la colaboración de organizaciones sin fines de lucro, convenios internacionales, etc. Un caso concreto es el Proyectos comunitarios orientados a sectores vulnerables pueden desarrollarse mediante fondo japonés, en el marco del Convenio de colaboración Asia-Pacífico ${ }^{2}$, programa que ha financiado la realización de más de 100 proyectos comunitarios en Chile. Otro caso concreto es el reportado en [5] donde se trabajó con la Oficina de ayuda a personas con discapacidad (OID) de la Municipalidad de Antofagasta. En este caso, se desarrolló un software para ayudar a niños con problemas del habla y lenguaje.

Con metodologías activas como Aprendizaje más Servicio $(A+S)$ es posible desarrollar en los alumnos las competencias relacionadas a proyectos software como: diseño e implementación de software con programación orientada a objetos y calidad software. También es posible desarrollar otras competencias como trabajo en grupo, comunicación oral y escrita y autogestión, todas estas necesarias para el correcto desarrollo profesional del estudiante del siglo XXI.

Experiencias como la reportada en [5] muestran que $\mathrm{A}+\mathrm{S}$ es una metodología que facilita el aprendizaje de técnicas de desarrollo software en asignaturas de la carrera ICI. Trabajos previos como [2, 3, 6] indican que los estudiantes, al terminar una carrera de ICI

2 https://www.bcn.cl/observatorio/asiapacifico/noticias/ proyecto-comunitario-apc-japon-chile y comenzar a trabajar, evidencian ciertas carencias en diseño e implementación de proyectos software.

En ese contexto, el trabajar con un proyecto real de corte solidario, puede facilitar la tarea de disminuir las brechas antes mencionadas, ya que los alumnos deben aplicar los principios de desarrollo de proyectos software y técnicas de programación de calidad, para alcanzar los objetivos planteados.

En este artículo se presenta una experiencia docente de aprendizaje activo [4] centrada en la mejora de técnicas de programación orientada a objetos y calidad software. En la asignatura se ha planteado un cambio en la estrategia de enseñanza, incorporando el compromiso comunitario al objetivo ya existente de crear un prototipo software con las características antes mencionadas. De forma concreta, se ha incorporado la metodología $\mathrm{A}+\mathrm{S}[5$, 12], con la participación de un socio comunitario que ha desempeñado el ron de cliente. El software se desarrolló para los juegos paralímpicos en la Región de Antofagasta, Chile. En el documento se describe la estrategia de enseñanza activa.

El resto de este documento está organizado de la forma siguiente: se describe después de la introducción el contexto de trabajo, detallando experiencias previas y metodologías activas, y concretamente $\mathrm{A}+\mathrm{S}$. Luego se detallan las características en la experiencia docente guiada por la estrategia de enseñanza activa [4]. Seguidamente se presenta la evaluación de la experiencia docente y las conclusiones. Posteriormente, se describen los agradecimientos y las referencias.

\section{CONTEXTO DE TRABAJO}

Las estrategias de aprendizaje juegan un rol importante en la adquisición de conocimiento [7]. De acuerdo con Eggen y Kauchak [8] las estrategias de aprendizaje son enfoques generales de herramientas pedagógicas para la enseñanza que se aplican en una variedad de áreas de contenido y se utilizan para alcanzar resultados de aprendizaje. Las estrategias de aprendizaje facilitan también el desarrollo de competencias es un contexto específico de trabajo. Experiencias recientes indican que el uso de metodologías activas en la estrategia docente facilita la adquisición de conocimiento, competencias [4] y desarrollo de competencias en proyectos software [9]. 
El proyecto educativo institucional (PEI) de la Universidad Católica del Norte $(\mathrm{UCN})^{3}[10]$ establece estrategias para el desarrollo de competencias en el aprendizaje, así como el fundamento de que el estudiante es responsable de su aprendizaje. En el PEI se declara, además, que la UCN está comprometida con el desarrollo profesional de los alumnos para que sean capaces de desarrollarse a nivel local, nacional e internacional e incluso que sean agentes de cambio social. En este contexto, se ha diseñado la estrategia de aprendizaje descrita a continuación.

En este trabajo la estrategia se ha desarrollado para la asignatura Taller de Programación (TP) de la carrera Ingeniería de Ejecución en Computación e Informática (IECI), carrera tecnológica que tiene declarado en su perfil de egreso ${ }^{4}$ un profesional integral y productivo, con principios éticos y con un sentido amplio de responsabilidad social, comprometido con el desarrollo regional y del país.

Esta asignatura se imparte en el V semestre de la carrera IECI. Los resultados de aprendizaje se detallan en la Tabla 1, junto con la relación que tienen con las herramientas pedagógicas que se describen a continuación:

- Clases magistrales, el docente entrega los contenidos del programa de la asignatura en dos bloques de clases a la semana. Estos contenidos se van entregando a medida que el proyecto solidario avanza y el objetivo subyacente es asegurar que los alumnos cuenten con bases teóricas para ir enfrentando los desafíos del proyecto software. Estos contenidos se dividen en cuatro unidades. También se incluyen en las clases los detalles de la metodología $\mathrm{A}+\mathrm{S}$ que se describe más adelante en la sección Metodologías activas.
Con esta herramienta se pretende trabajar y desarrollar las competencias relativas a la programación orientada a objetos y técnicas para medir la calidad del producto software.

- Entrevistas con el cliente, el docente planifica entrevistas periódicas con el socio comunitario. La primera entrevista se realiza para conocer el socio comunitario, su trabajo e identificar el problema o necesidad de un producto software. Las siguientes entrevistas sirven para afinar la comprensión de la necesidad de un producto software, y verificar los avances y/o resultados intermedios en el proyecto. Las entrevistas próximas al final del semestre sirven para validar los resultados del proyecto y conocer la satisfacción del cliente con dicho producto. Para la segunda y tercera tanda de entrevistas, los alumnos pueden participar en la re-planificación (si fuera necesario). Con esta herramienta se pretende trabajar competencias de trabajo en grupo, comunicación y autogestión, a parte de las disciplinares.

- Desarrollo del proyecto, el docente guía el desarrollo del proyecto software que consiste en aplicar etapas de un proyecto software (análisis, diseño, implementación, pruebas y mantenimiento) junto a la programación orientada para lograr el producto software. Con esta herramienta se pretende trabajar todas las competencias mencionadas anteriormente.

- Presentaciones orales, los alumnos realizan presentaciones orales de avances en el proyecto, frente al docente, socio comunitario y personas relacionadas/interesadas (otros profesores, etc.). En estas presentaciones los alumnos expresan los resultados en el proyecto y desarrollan también competencias relativas a la comunicación.

Tabla 1. Relación entre objetivos de aprendizaje y herramientas pedagógicas en el trabajo.

\begin{tabular}{|l|c|c|c|c|}
\cline { 2 - 5 } \multicolumn{1}{c|}{} & $\begin{array}{c}\text { Clases } \\
\text { magistrales }\end{array}$ & $\begin{array}{c}\text { Entrevistas } \\
\text { con el cliente }\end{array}$ & $\begin{array}{c}\text { Trabajo con } \\
\text { proyecto }\end{array}$ & $\begin{array}{c}\text { Presentaciones } \\
\text { orales }\end{array}$ \\
\hline Desarrollo de proyectos software & $\mathrm{X}$ & $\mathrm{X}$ & $\mathrm{X}$ & $\mathrm{X}$ \\
\hline Técnicas de programación orientada a objetos & $\mathrm{X}$ & & $\mathrm{X}$ & $\mathrm{X}$ \\
\hline Calidad del producto software & $\mathrm{X}$ & $\mathrm{X}$ & $\mathrm{X}$ & $\mathrm{X}$ \\
\hline Técnicas de mantenimiento de desarrollo software & $\mathrm{X}$ & $\mathrm{X}$ & $\mathrm{X}$ & $\mathrm{X}$ \\
\hline
\end{tabular}

3 https://www.ucn.cl/sobre-ucn/somos-ucn/proyecto-educativo-ucn/

4 http://www.disc.ucn.cl/ingenieria - en- computacion-e-informatica/ 


\section{Metodologías activas}

En la docencia relacionada con carreras de ICI, las metodologías activas han demostrado ser un medio importante para alcanzar aprendizaje significativo, desarrollo de competencias $[1,16$, 17]. En las metodologías activas, el aprendizaje se alcanza mediante un proceso participativo donde el estudiante es parte activa y responsable de su propio aprendizaje $[1,2,18]$.

También, el aprendizaje es enfocado como un proceso constructivo y no receptivo, en el cual el estudiante tiene la oportunidad de investigar, experimentar, interactuar, reflexionar y comunicarse tanto con el docente como con el resto de la clase. En este esquema de trabajo el docente cumple el rol de facilitador del proceso, todo esto, preferiblemente, en el contexto de enfrentar problemas del mundo real o de la práctica profesional $[8,12]$.

En este trabajo se seleccionó $\mathrm{A}+\mathrm{S}$ como marco activo de trabajo para facilitar el logro de los resultados de aprendizaje. A+S es descrita en trabajos como [19, 20] como una metodología pedagógica experiencial, que se caracteriza por la integración de actividades de servicio a la comunidad en el currículo académico, donde los alumnos utilizan los contenidos y las herramientas académicas en atención a necesidades genuinas de una comunidad. Este contexto de trabajo está integrado por un socio comunitario (cliente) y el equipo de trabajo: alumnos y docentes. El socio comunitario es cualquier institución u asociación que aporte a la comunidad en bienes o servicios. En general, las etapas de esta metodología son:

- Selección del socio comunitario, en esta etapa se prepara la propuesta de proyecto orientado a cubrir las necesidades de una comunidad. En el caso particular de a asignatura, el socio comunitario debe tener la necesidad de un proyecto software.

- Desarrollo del proyecto, igualmente en el caso concreto, consta del desarrollo de un proyecto software para un socio comunitario.

- Evaluación de resultados, en esta etapa se valida la utilidad práctica del proyecto comunitario (en este caso proyecto software) y su impacto en beneficio de la comunidad.

\section{Experiencias previas}

Existen trabajos previos que han reportado el uso de metodologías activas para mejorar el aprendizaje de conceptos de Ingeniería, concretamente de
Ingeniería de Software y desarrollo de Proyectos Software. Por ejemplo, en [5] se describe un trabajo previo con $\mathrm{A}+\mathrm{S}$ y desarrollo de un proyecto software para facilitar la formación de frases en terapia del lenguaje con niños con dificultades de este tipo, esta experiencia docente permitió identificar las buenas prácticas para dar base a lo reportado en este documento. También en [11] se describe un trabajo hecho en la Universidad Nacional de Tres de Febrero, Argentina, donde se adoptó un enfoque del aula invertida combinado con otras técnicas de enseñanza no tradicionales para un curso de Ingeniería de Software.

En [2] se reporta un estudio realizado en dos universidades de Chile para la asignatura Ingeniería del Software, la estrategia de aprendizaje en este caso consistió en la combinación de metodologías activas de aprendizaje y métodos ágiles de desarrollo de sistemas software en un marco común de trabajo (framework). Las pedagogías incluidas en ese framework fueron aprendizaje basado en problemas y metodologías ágiles de desarrollo software ${ }^{5}$. Esta estrategia permitió a los estudiantes adquirir un conocimiento más profundo y aplicarlo en una forma práctica, además los estudiantes asumieron roles de proyectos reales (líder de proyecto, programador, probador, etc.).

En [3] se describe una estrategia de enseñanza orientada a desarrollar competencias y puesta en práctica de conceptos relativos a la Ingeniería de Software en un curso de postgrado. La estrategia consta de clase invertida y actividades que acercan al estudiante a la práctica profesional.

En otros trabajos como $[13,14]$ han usado PBL (Problem-Based Learning) junto con principios de $\mathrm{SCRUM}^{6}$, como parte de la estrategia de estudio en el desarrollo de proyectos software. Los resultados en estos proyectos han sido satisfactorios particularmente en el uso de metodologías, cumplimiento de plazos y la usabilidad del producto final. En [15] se describe también un trabajo con metodologías activas y SCRUM para desarrollar aplicaciones móviles, con participación activa durante todo el proceso de actores

\footnotetext{
5 https://danielgrifol.es/metodologias-agiles-de -desarrollo-de-software/

6 https://www.scrum.org/
} 
como el cliente y otros relacionados/interesados en el desarrollo (los denominados stakeholders).

\section{DESCRIPCIÓN DE LA EXPERIENCIA DOCENTE}

En este apartado se describe la planificación hecha para el logro de aprendizaje significativo según los resultados de aprendizaje ya descritos. La estrategia de enseñanza activa consistió en combinar principios de desarrollo de proyectos software basado en etapas de SCRUM, con las etapas de A+S.

El socio comunitario en este proyecto fue la Oficina para integración de personas con Discapacidad (OID) de Antofagasta, dependiente de la Ilustre Municipalidad de Antofagasta. El grupo de alumnos fue de 10 , repartidos en grupos de trabajo de 2 alumnos, cada grupo asumía tareas que aportaban al proyecto y debían coordinarse con el resto de grupos para asegurar el avance del proyecto en la dirección correcta. Los objetivos específicos en esta experiencia son:

1. Utilizar las etapas de desarrollo de proyecto software y técnicas de programación orientada a objetos, para guiar la construcción de una herramienta software tipo $\mathrm{APP}^{7}$ destinada a cubrir una necesidad del socio comunitario, en el marco de un proyecto solidario.

2. Utilizar técnicas de validación y pruebas de aplicaciones software tipo APP, para asegurar la calidad del producto software según las necesidades del socio comunitario.

3. Utilizar técnicas y principios de Ingeniería del Software para generar un plan de mantenimiento del prototipo software que asegure la usabilidad del producto en un tiempo cercano a 5 años.

Para alcanzar los objetivos antes descritos se diseñó la estrategia docente con las siguientes características:

- En las clases se dictaron los contenidos programáticos y se realizaron también evaluaciones escritas dirigidas a medir la comprensión de los conceptos y también verificar la aplicabilidad que estaban dando los alumnos a dichos conceptos en el desarrollo del proyecto software. Estas evaluaciones tenían una ponderación del $40 \%$ de la nota de la asignatura, se realizaron 2 evaluaciones escritas con ponderación de $15 \%$

\footnotetext{
7 https://www.qode.pro/blog/que-es-una-app/
}

cada una y 2 evaluaciones cortas con un peso de $5 \%$ cada una. Algunas de estas clases fueron dedicadas a trabajar con el código software, sobre todo en la segunda mitad del proyecto.

- Para el trabajo con el proyecto se cubrieron etapas típicas de proyectos software como son: (a) definición de requerimientos, (b) análisis y diseño, (c) programación, (d) validación de código software y (e) diseño de la etapa de mantenimiento. Para las etapas (a) y (b) se realizaron entrevistas con el socio comunitario al inicio del proyecto, para las etapas (c) y (d) se realizaron clases de ayudantía (clases prácticas donde un alumno de cursos más avanzados, que ya ha cursado la asignatura y con amplios conocimiento $\mathrm{s}$ en programación orientada a objetos, ayuda y guía la codificación software). El proyecto software tenía una ponderación del $40 \%$ de la nota de la asignatura, se realizaron tres evaluaciones de proyecto (mediante presentación oral), las dos primeras con un peso de $15 \%$ cada una y la final con un peso del $10 \%$.

En detalle, las presentaciones orales estuvieron orientadas a mostrar avances por parte de los alumnos, en el desarrollo del proyecto software. En las presentaciones orales participó activamente el socio comunitario (cliente) con dos roles principales: dador y validador de la información necesaria para generar el proyecto software, y también como evaluador de los resultados. En las presentaciones, el cliente tuvo un 5\% en la primera y segunda, y un $10 \%$ en la tercera presentación.

Para estas presentaciones se generó una rúbrica de evaluación con objetivo en verificar: calidad de los requisitos (forma de escribir, nivel de validación con el cliente, etc.), cumplimiento de las necesidades del cliente (descritas en el documento de requisitos) y calidad del producto software.

Con lo requerido para evaluar y las ponderaciones, los alumnos gestionaron las presentaciones, definiendo los contenidos de cada una de ellas en función a lo visto en clase y a los avances alcanzados en cada etapa o iteración del desarrollo software.

Para validar el logro del trabajo en equipo, se diseñó un instrumento con 3 preguntas, que fue usado por los alumnos en la última presentación, para 
evaluar a cada compañero de su equipo de trabajo. El instrumento se usó de la forma siguiente:

- Cada alumno evaluaba individualmente a cada uno de sus compañeros de grupo. Con las preguntas y según la forma que se detalla en la Tabla 2.

- Una vez obtenidas las respuestas y la nota de cada alumno, se promediaron las notas parciales para obtener una de la percepción del resto de los compañeros para cada uno de ellos.

Esta evaluación se realizó en la última presentación y formó parte de la nota de esa última presentación, con una ponderación del 10\% de esa nota.

\section{EVALUACIÓN}

La evaluación en esta innovación se ha dirigido en dos aspectos principales: valorar el logro de los resultados de aprendizaje de la asignatura y valorar el logro de competencias de comunicación y trabajo en grupo. Ambos aspectos se han medido respecto a los resultados en las evaluaciones escritas y en el proyecto.

La importancia pedagógica de aprender a través de desarrollo de proyectos (PBL-Project-Based learning) es destacada en trabajos previos como [13, 21]. También trabajar con $\mathrm{A}+\mathrm{S}$ es una práctica $\mathrm{PBL}$ con proyecto solidario, orientado a la comunidad, y es precisamente este tipo de trabajo (solidario) que ha motivado a los alumnos a participar en más del $85 \%$ de las actividades programadas.

Las calificaciones es la forma de medir el logro de los objetivos, como muestra la Figura 1, se han considerado 7 rangos de notas: A para las notas entre 1 y 3.9, B para las notas entre 4.0 y $4.5, \mathrm{C}$ para las notas entre 4.6 y 5 ,

Tabla 2. Preguntas usadas en el instrumento evaluación de los compañeros. La escala de evaluación en Chile es de 1 a 7 puntos (mínima aprobatoria es 4.0).

\begin{tabular}{|c|c|c|c|c|}
\hline $\begin{array}{l}\text { El alumno mostró dis- } \\
\text { posición al logro con } \\
\text { aportes significativos }\end{array}$ & $\begin{array}{l}\text { En el } 100 \text { de los casos } \\
\text { Nota (entre } 7 \text { y } 6.1 \text { ) }\end{array}$ & $\begin{array}{l}\text { En más del } 70 \% \text { de } \\
\text { los casos } \\
\text { Nota }(\text { entre } 6 \text { y } 5.1)\end{array}$ & $\begin{array}{l}\text { En más del } 50 \% \text { de } \\
\text { los casos } \\
\text { Nota (entre } 5 \text { y } 4.0 \text { ) }\end{array}$ & $\begin{array}{l}\text { En menos del } 50 \% \text { de } \\
\text { los casos } \\
\text { Nota (entre } 3.9 \text { y } 1 \text { ) }\end{array}$ \\
\hline $\begin{array}{l}\text { El alumno participó acti- } \\
\text { vamente en las reuniones } \\
\text { de trabajo en grupo }\end{array}$ & $\begin{array}{l}\text { En el } 100 \text { de los casos } \\
\text { Nota (entre } 7 \text { y } 6.1 \text { ) }\end{array}$ & $\begin{array}{l}\text { En más del } 70 \% \text { de } \\
\text { los casos } \\
\text { Nota (entre } 6 \text { y } 5.1 \text { ) }\end{array}$ & $\begin{array}{l}\text { En más del } 50 \% \text { de } \\
\text { los casos } \\
\text { Nota (entre } 5 \text { y } 4.0 \text { ) }\end{array}$ & $\begin{array}{l}\text { En menos del } 50 \% \text { de } \\
\text { los casos } \\
\text { Nota (entre } 3.9 \text { y } 1)\end{array}$ \\
\hline $\begin{array}{l}\text { El alumno aportó su tra- } \\
\text { bajo individual para el } \\
\text { logro del proyecto }\end{array}$ & $\begin{array}{l}\text { En el } 100 \text { de los casos } \\
\text { Nota (entre } 7 \text { y } 6.1 \text { ) }\end{array}$ & $\begin{array}{l}\text { En más del } 70 \% \text { de } \\
\text { los casos } \\
\text { Nota (entre } 6 \text { y } 5.1 \text { ) }\end{array}$ & $\begin{array}{l}\text { En más del } 50 \% \text { de } \\
\text { los casos } \\
\text { Nota (entre } 5 \text { y } 4.0 \text { ) }\end{array}$ & $\begin{array}{l}\text { En menos del } 50 \% \text { de } \\
\text { los casos } \\
\text { Nota (entre } 3.9 \text { y } 1 \text { ) }\end{array}$ \\
\hline
\end{tabular}

\section{Relación porcentajes de aprobación en el curso}

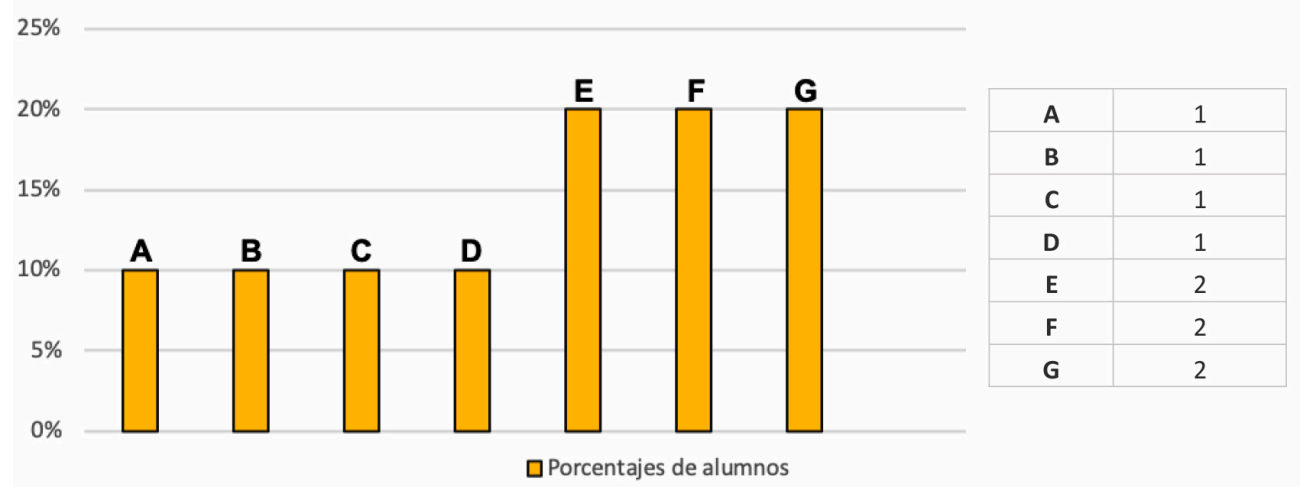

Figura 1. Relación de alumnos por rango de aprobación en la asignatura TP. 
D para las notas entre 5.1 y 5.5, E para las notas entre 5.6 y $6.0, \mathrm{~F}$ para las notas entre 6.1 y $6.5, \mathrm{G}$ para las notas entre 6.6 y 7.0. Como se puede observar en la figura 1, solo el $10 \%$ de los alumnos está por debajo del 4.0 , el $30 \%$ de los alumnos se encuentra entre el 4 y 5.5 y el $60 \%$ con notas superiores al 5.5, lo que se puede interpretar como que el rendimiento, logro de los objetivos de aprendizaje, fue alcanzado de forma muy satisfactoria. En base a estos resultados se considera logrado el cumplimiento de los tres primeros objetivos de aprendizaje.

También los resultados de la valoración del trabajo en grupo fueron, dado que el $90 \%$ de los alumnos participó en el 70\% o más de las actividades programadas y realizaron aportes significativos para el logro del proyecto. Con estos resultados se considera logrado el cuarto objetivo de aprendizaje.

\section{CONCLUSIONES}

Como conclusiones se destaca lo siguiente:

- La estrategia de enseñanza ha motivado la participación activa de los alumnos, desde el principio hasta el final del curso. La mayor parte de los alumnos ha cumplido con las tareas y actividades programadas según las etapas de desarrollo de proyecto software.

- Los alumnos han trabajado con interés en todas las actividades según las herramientas pedagógicas. Es destacable la interacción lograda con el socio comunitario, que fue inspirador para el cumplimiento de los plazos de entrega.

- La innovación dirigida por la estrategia de enseñanza ha permitido poner en práctica las técnicas de buena programación en el marco de un desarrollo ágil, con entregas frecuentes y feedback, también frecuente, de parte del socio comunitario. Esto acerca a los alumnos a una práctica real de entorno de desarrollo software.

- $\mathrm{Al}$ ser un desarrollo real y para un cliente real, los alumnos se han esforzado por generar un producto (prototipo) validado.

- La interacción frecuente (mediante reuniones e incluso trabajo conjunto) con el socio comunitario y su participación en las evaluaciones del proyecto, fueron elementos decisivos en la calidad del prototipo final del producto software.

- La evaluación del socio comunitario del producto mediante un porcentaje en la nota ha logrado inspirar el trabajo de calidad en los alumnos.
Como conclusiones también se puede destacar lo siguiente, este tipo de estrategia docente ha facilitado la labor de enseñar del docente y ha permitido un acercamiento al rol de facilitador del proceso de aprendizaje de los alumnos. También ha motivado la auto gestión en el aprendizaje de nuevas técnicas de programación, de integración de herramientas CASE al ambiente de desarrollo por parte de los alumnos. La experiencia para el docente ha sido muy motivadora $\mathrm{y}$ con resultados muy satisfactorios desde el punto de vista de experiencia real de desarrollo de un producto software de utilidad para la comunidad de la OID.

La Figura 1 detalla los grupos y cantidad de alumnos por grupo que se han usado en la validación. El gráfico de la parte izquierda ubica a cada uno de los rangos (desde el A hasta G) de forma porcentual, según la cantidad de alumnos que hay en dicho rango. El detalle de cantidad de alumnos en cada uno de los rangos está descrito en la parte derecha de la figura. Por ejemplo, en el grupo E hay dos alumnos y este grupo representa el 20\% del 100\% total de los alumnos en el curso TP.

Otra conclusión interesante es que la estrategia basada en combinar etapas de la metodología activa $\mathrm{A}+\mathrm{S}$ y actividades basadas en el desarrollo ágil, y la orientación a un proyecto enmarcado en la responsabilidad social, ha motivado a los alumnos a participar durante toda la asignatura, dado que los alumnos se han sentido identificados con una problemática a resolver y han visto el aporte real a la OID.

Como posibles líneas futuras de trabajo se pueden destacar las siguientes:

- Incorporar esta forma de trabajo en asignaturas cercanas al desarrollo de proyectos software, como por ejemplo Ingeniería del Software o Taller de Sistemas (asignatura que tiene contenidos de Planificación de Sistemas Software).

- Intentar aplicar esta misma forma de trabajo, pero con un proyecto de empresa, para tratar de identificar similitudes o diferencias en la forma de desarrollar el trabajo, evaluar resultados y evaluar la participación e interés de los estudiantes.

En este trabajo, los indicadores principales para medir resultados han sido la nota de los estudiantes 
y la participación en las actividades. Se pretende $\mathrm{n}$ el futuro incorporar otros indicadores, como por ejemplo la valoración del estudiante de lo que espera desarrollar en el curso y lo que realmente ha logrado. Esto permitirá tener una valoración más objetiva de la experiencia docente.

\section{AGRADECIMIENTOS}

El autor agradece la colaboración del personal de la Oficina para la Integración de personas con Discapacidad OID de Antofagasta por el apoyo prestado al logro de este proyecto, especialmente agradecer a Paola Selti, quien ha sido la cara visible del cliente, usuario y un miembro más del equipo de trabajo, para lograr que este proyecto piloto haya tenido un excelente resultado en este curso académico.

\section{REFERENCIAS}

[1] V. Flores, P. Lara and MC. Gaya. "Project Based Engineering School, Una escuela conectada con un mundo profesional sostenible". X Jornadas Universitarias de Innovación Universitaria (JENUI), pp. 476484. 2013.

[2] V. Flores and J. Gomez. "Applying active methodologies for teaching software engineering in computer engineering". IEEE Revista Iberoamericana de Tecnologías del Aprendizaje. Vol. 12 N $^{\circ}$ 4, pp. 182-190. DOI: 10.1109/RITA.2017.2778358.

[3] J. Joy and V.G. Renumol. "Active oriented teaching strategy for Software Engineering: An experience". Journal of Information Technology Education: Innovations in Practice. Vol. 17, pp. 181-200. 2018. DOI: $10.28945 / 4116$.

[4] Y. Sedelmaier and D. Landes. "Active and Inductive Learning in Software Engineering Education. In 2015 IEEE/ACM 37th IEEE International Conference on Software Engineering, pp. 418-427. 2015.

[5] V. Flores and C. Lepe. "Using A+ S and agile development to achieve meaningful learning in teaching software projects". In 36th International Conference of the Chilean Computer Science Society (SCCC), pp. 1-6. 2017. IEEE. DOI: 10.1109/ SCCC.2017.8405109.
[6] S.B. Cooper, J.H. Cripps and J.I. Reisman. "Service-learning in deaf studies: Impact on the development of altruistic behaviors and social justice concern". American Annals of the Deaf. Vol. 157 N $^{\circ}$ 5, pp. 413-427. 2012. DOI: 10.1353/aad.2013.0003.

[7] C.E. Weinstein, T.W. Acee and J. Jung. "Self-regulation and learning strategies". New Directions for Teaching and Learning. Vol. 126, pp. 45-53. 2011. DOI: 10.1002/ tl. 443

[8] P.D. Eggen, D.P. Kauchak. "Strategies and Models for Teachers". Teaching Content and Thinking Skills. Pearson Education Inc. 2006.

[9] J.S. Jeong, D. González-Gómez, A. GallegoPicó and J.C. Bravo. "Effects of active learning methodologies on the students' emotions, self-efficacy beliefs and learning outcomes in a science distance learning course". Journal of Technology and Science Education. Vol. 9, pp. 217-227. 2019. DOI: 10.3926/jotse.530.

[10] UCN. "Proyecto Educativo Institucional". Universidad Católica del Norte. Antofagasta, Chile. 2017.

[11] N.M. Paez. "A flipped classroom experience teaching software engineering". In the 1st International Workshop on Software Engineering Curricula for Millennials, pp. 16-20. 2017.

[12] S.B. Cooper, J.H. Cripps and J.I. Reisman. "Service-learning in deaf studies: Impact on the development of altruistic behaviors and social justice concern". American Annals of the Deaf. Vol. 157 No 5, pp. 413-427. 2012.

[13] D.G. Lamar, P.F. Miaja, M. Arias, A. Rodríguez, M. Rodríguez, A. Vázquez and J. Sebastián. "Experiences in the application of project-based learning in a switching-mode power supplies course". IEEE Transactions on Education. Vol. 55, pp. 69-77. 2012.

[14] M. Singh. "U-SCRUM: An agile methodology for promoting usability”. In Agile, AGILE'08, pp. 555-560. 2008.

[15] A.R. Sales, A. Fernandez and M. Nichols. "Combining challenge-based learning and scrum framework for mobile application development". In Proceedings of the 2015 ACM Conference on Innovation and Technology in Computer Science Education, pp. 189-194. 2015. 
[16] C. Tosun and C. Senocak. "The effects of problem-based learning on metacognitive awareness and attitudes toward chemistry of prospective teachers with different academic backgrounds". Australian Journal of Teacher Education. Vol. $38 \mathrm{~N}^{\mathrm{o}}$ 3, pp. 4-8. 2013.

[17] S.B. Cooper, J.H. Cripps and J.I. Reisman. "Service-learning in deaf studies: Impact on the development of altruistic behaviors and social justice concern". American Annals of the Deaf. Vol. $157 \mathrm{~N}^{\circ}$ 5, pp. 413-427. 2012.

[18] S. Bell. "Project-based learning for the 21st century: Skills for the future". The Clearing House, Vol. 83 No 2, pp. 39-43. 2010.

[19] Ch. Jouannet, M.H. Salas and M.A. Contreras. "Modelo de implementación de Aprendizaje Servicio $(\mathrm{A}+\mathrm{S})$ en la UC. Una experiencia que impacta positivamente en la formación profesional integral". Revista Calidad en la Educación. Vol. 13, pp. 197-212.

[20] S.B. Cooper, J.H. Cripps and J.I. Reisman. "Service-learning in deaf studies: Impact on the development of altruistic behaviors and social justice concern". American Annals of the Deaf. Vol. 157, pp. 413-427. 2012.

[21] P.C. Blumenfeld, E. Soloway, R.W. Marx, J.S. Krajcik, M. Guzdi-al and A. Palincsar. "Motivating project-based learning: Sustaining the doing supporting the learning". Educational Psychologist. Vol. 26, pp. 369398. 1991. 